\title{
Light quark mass effects in Higgs boson production in gluon fusion
}

\author{
Alexander Penin** \\ Department of Physics, University of Alberta, Edmonton, Alberta T6G 2J1, Canada, and \\ Institut für Theoretische Teilchenphysik, KIT, 76128 Karlsruhe, Germany \\ E-mail: penin@ualberta.ca
}

Production of Higgs bosons at the LHC is affected by the contribution of light quarks, that mediate the $\mathrm{gg} \rightarrow \mathrm{Hg}$ transition. Although their impact is suppressed by small Yukawa couplings, it is enhanced by large logarithms of the ratio of the Higgs boson mass or its transverse momentum to light quark masses. We study the abelian corrections to $\mathrm{gg} \rightarrow \mathrm{Hg}$ amplitudes of the form $\left(C_{F} \alpha_{s} \mathscr{L}^{2}\right)^{n}$, where $\mathscr{L} \in\left\{\ln \left(s / m_{b}^{2}\right), \ln \left(p_{\perp}^{2} / m_{b}^{2}\right)\right\}$ and show how these non-Sudakov double logarithmic terms can be resummed to all orders in the strong coupling constant.

Loops and Legs in Quantum Field Theory

24-29 April 2016

Leipzig, Germany

* Speaker.

${ }^{\dagger}$ The talk is based on Ref. [1] 


\section{Introduction}

The Higgs boson discovered at the LHC by ATLAS and CMS collaborations almost four years ago $[2,3]$ is a mysterious particle. Indeed, it seems to fit perfectly into the Standard Model (SM) of particle physics and its mass is numerically close to the weak scale $v$. However, the mechanism that would tie these two quantities together in a more general theory requires presence of other, relatively light, particles that couple to the Higgs boson. Such particles have not been observed so far and limits on their masses gradually become so tight that the "natural" relation $m_{H} \sim v$ is endangered. Further exploration of Higgs boson properties, including its couplings and quantum numbers, will be essential for understanding to what extent the observed particle is indeed described by the Standard Model and, hopefully, for discovering clues as to what the mass scale of physics beyond the Standard Model can actually be.

An important observable in Higgs physics is the Higgs boson transverse momentum distribution. There are several reasons for that. On one hand, precise knowledge of the Higgs boson $p_{\perp}$-distribution is important for understanding jet-vetoed cross sections and, more generally, observables subject to experimental constraints. The uncertainties in modeling the $p_{\perp}$-distribution affect values of the Higgs coupling constants extracted from such fiducial quantities. Since the total inclusive Higgs boson production cross section is currently known through next-to-next-tonext-to-leading order in perturbative QCD [4], the uncertainty in the Higgs $p_{\perp}$-distribution may become the dominant one when future experimental data is confronted with theoretical predictions for the Higgs boson production.

Further motivation for the precise description of the Higgs boson $p_{\perp}$-spectrum comes from the observation that the $p_{\perp}$-distribution is, potentially, a good observable for detecting relatively light $\left(m \sim m_{H}\right)$ colored particles that couple to the Higgs boson [5]. Indeed the contribution of a particle with the mass $m \sim m_{H}$ to the Higgs boson production in gluon fusion is almost independent of $p_{\perp}$ for $p_{\perp}<m$ while for $p_{\perp}>m$ it rapidly decreases. Thus the $p_{\perp}$-distribution of Higgs bosons or jets recoiling against it, may serve as a sensitive probe of this type of physics beyond the Standard Model.

High-precision theoretical predictions for Higgs boson $p_{\perp}$-distribution within the Standard Model are necesary to pursue this program $[6,7,8,9]$. Unfortunately, despite significant progress in understanding the Higgs $p_{\perp}$-spectrum in recent years, the overall situation is unsatisfactory. The main challenge is to describe the bottom quark contribution to the Higgs boson production in gluon fusion at moderate values of the transverse momentum. Indeed, the $g g \rightarrow H$ transition in the Standard Model is dominated by the top-quark loop, thanks to the large Higgs-top Yukawa coupling. Since the top quark mass is large compared to the Higgs mass, it is possible to integrate out the top quark and describe the Higgs production at sufficiently low transverse momentum in the effective field theory with a local $g g H$ interaction. This reduces the number of loops in perturbative computations by one and allows us to push them to very high orders in QCD perturbation theory. Within this approximation, the Higgs $p_{\perp}$-distribution has been evaluated through next-to-next-toleading order at high $p_{\perp}<m_{t}[10,11]$ and to next-to-next-to-leading logarithmic accuracy at low $p_{\perp}<m_{H}[12,13] .{ }^{1}$

\footnotetext{
${ }^{1}$ For a recent discussion and further references see Ref. [14].
} 
At the same time understanding the bottom-quark contribution to $g g \rightarrow H g$ turned out to be more involved. ${ }^{2}$ Indeed, since $m_{b} \sim 4.2 \mathrm{GeV}$, the point-like approximation for the bottom quark contribution to $g g H$ vertex is only valid for tiny transverse momenta $p_{\perp}<m_{b}$. In a broader and more interesting momentum region $p_{\perp}>m_{b}$, the local vertex approximation for the bottom quarkmediated $g g H$ interaction is invalid and we must deal with the computation of complicated box diagrams with internal masses. Calculation of such diagrams at two and more loops is beyond the reach of existing computational techniques. ${ }^{3}$ As the result, the $g g \rightarrow H g$ amplitudes for $p_{\perp}>m_{b}$ are only known in the leading (one-loop) approximation.

The bottom quark contribution to Higgs boson production is small, compared to the contribution of the top quark. However, it is still relevant phenomenologically because of the high precision of forthcoming experimental measurements of the Higgs-gluon coupling and because the bottom quark contribution is dynamically enhanced. Indeed, although the coupling of the bottom quark to the Higgs boson is small compared to the Higgs-top coupling, the $n$-loop bottom quark contribution to $g g \rightarrow H g$ is enhanced by two powers of large logarithms per one power of $\alpha_{s}$, i.e. $\mathscr{O}\left(\alpha_{s}^{n} \mathscr{L}^{2 n}\right)$, where $\mathscr{L} \in\left\{\ln \left(m_{H}^{2} / m_{b}^{2}\right), \ln \left(p_{\perp}^{2} / m_{b}^{2}\right)\right\}$. For relevant values of the transverse momentum $p_{\perp} \sim 30 \mathrm{GeV}$ and the Higgs boson mass $m_{H}=125 \mathrm{GeV}$, these logarithms can be numerically quite large $\mathscr{L}^{2} \sim 20-50$. In fact, the magnitude of the double logarithmic corrections suggests that the all-order resummation may be necessary.

The origin of these logarithmically enhanced terms is currently not well understood. Although their double logarithmic nature suggests a mechanism similar to the Sudakov enhancement [18], as we explain below the mass suppression of the amplitude $\mathscr{M}_{g g \rightarrow H g} \sim m_{b}^{2}$ makes such an interpretation problematic. Contribution of bottom quarks to the Higgs boson production in gluon fusion was discussed in Refs. $[19,20,21]$ in the context of $p_{\perp}$-resummation. There it was pointed out that the standard technology of $p_{\perp}$-resummation only applies for $p_{\perp}<m_{b}$, while for larger values of $p_{\perp}$ it is incomplete. The authors of Refs. [19, 20,21] then used differences between various resummation prescriptions to estimate the uncertainty in the Higgs $p_{\perp}$-distribution, caused by unknown higher-order QCD corrections to the bottom quark contribution.

The goal of this paper is to make a step towards a better understanding of the origin of double logarithmic corrections to the Higgs boson production, their computation in the two-loop approximation and to their resummation. Since these tasks are very challenging, we restrict our analysis to abelian QCD corrections, i.e. corrections associated with the abelian color factor $C_{F}^{n}$ in the $n$-th order of QCD perturbation theory. Note that the abelian radiative corrections are generated by the coupling of virtual gluons to massive off-shell quarks. As a consequence, these corrections are infra-red finite on their own, so that physical results can be obtained without the need to consider processes with additional soft and collinear radiation.

\section{The approximation for the bottom quark contribution to $g g \rightarrow H g$ amplitudes}

We consider the Higgs boson production in the process $g g \rightarrow H g$ mediated by the bottom quark loop. The Higgs boson has a non-vanishing transverse momentum. The particle momenta

\footnotetext{
${ }^{2}$ Contributions of even lighter quarks are negligible.

${ }^{3}$ For a recent progress in the order analysis of the bottom quark mass effects in $g g \rightarrow H$ production see Refs. [15, $16,17]$.
} 


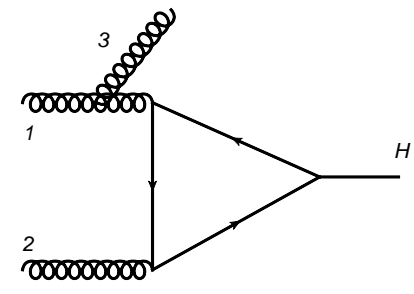

a)

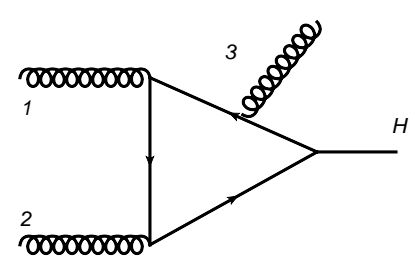

b)

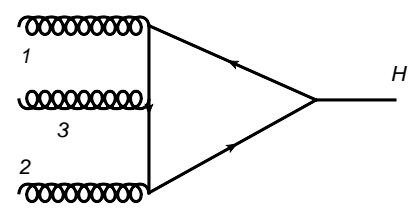

c)

Figure 1: One-loop diagrams representing the leading order bottom quark contribution to $g g \rightarrow H g$ process. Symmetric diagrams corresponding to the opposite direction of the quark flow and to the soft emissions off the opposite gluon/quark line are not shown.

are assigned in the following way

$$
g\left(p_{1}\right)+g\left(p_{2}\right) \rightarrow g\left(p_{3}\right)+H\left(p_{H}\right) .
$$

Our goal is to find the double logarithmic contributions to helicity amplitudes in a kinematic situation where the energy of the final state gluon $E_{3}$ is much smaller than the energies of the colliding gluons $E_{1,2}$ and the Higgs boson mass. At the same time, we consider $E_{3}$ to be much larger than the mass of the quark that mediates the $g g \rightarrow H$ transition. When written in terms of kinematic invariants, these conditions become

$$
m_{b}^{2} \ll t, u \ll s, m_{H}^{2}
$$

where $s=\left(p_{1}+p_{2}\right)^{2}, t=\left(p_{1}-p_{3}\right)^{2}, u=\left(p_{2}-p_{3}\right)^{2}$. Eq.(2.2) in particular implies that $m_{b}^{2} \ll p_{\perp}^{2} \ll$ $t, u$, where $p_{\perp}^{2}=t u / s$ is the square of the transverse momentum of the Higgs boson or the gluon in the final state.

To illustrate this kinematic situation further, consider the production of a Higgs boson through a bottom quark loop accompanied by an emission of a soft gluon. We take $m_{b}=4.2 \mathrm{GeV}, \sqrt{s} \approx m_{H}$, $p_{\perp} \approx 20 \mathrm{GeV}$ and assume central production (small rapidity), so that $E_{3} \approx p_{\perp}$. Numerically we find

$$
\frac{m_{b}}{E_{3}} \sim \lambda, \quad \frac{E_{3}}{E_{1}} \sim \lambda,
$$

with $\lambda \sim 0.25$. We consider $\lambda$ to be a small parameter and adopt the scaling rules Eq.(2.3). In the limit $\lambda \rightarrow 0$ the $g g \rightarrow H g$ amplitude develops the $1 / \lambda$ singularity, characteristic to the soft gluon emissions; this allows us to write the perturbative series for the amplitude in the following way

$$
\mathscr{M}_{g g \rightarrow H g}=\frac{g_{s}}{\lambda} \sum_{n=1}^{\infty} C_{n} \alpha_{s}^{n} \ln ^{2 n}(\lambda)+\ldots
$$

In Eq.(2.4), we neglected all terms that are less singular than $\lambda^{-1} \alpha_{s}^{n} \ln ^{2 n} \lambda$ in the $\lambda \rightarrow 0$ limit. We are interested in the abelian part of the coefficients $C_{n}$, which determine the double logarithmic approximation for the amplitude. The double logarithmic corrections in Eq.(2.4) are generated by the soft quark exchange rather than the soft gluon exchange responsible for the well known Sudakov double logarithms. The structure of the non-Sudakov logarithmic corrections characteristic to the 


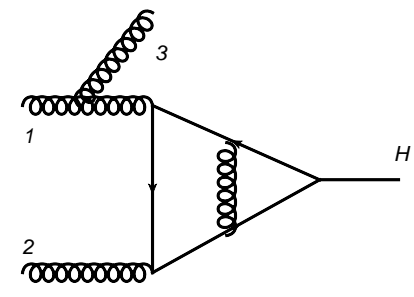

a)

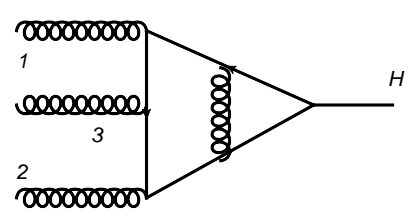

d)

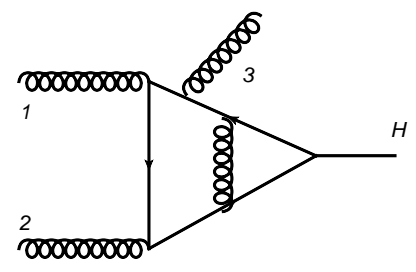

b)

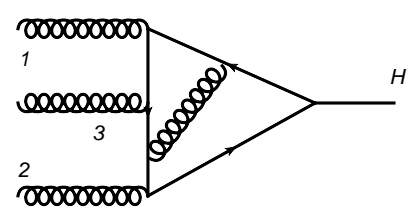

e)

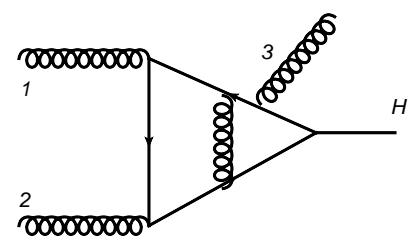

c)

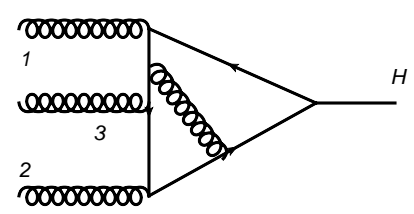

f)

Figure 2: Two-loop diagrams contributing to the abelian double logarithmic corrections. Diagrams that differ by the direction of the fermion flow are not shown.

mass suppressed amplitudes is much more complicated [22, 23, 24]. In particular, in contrast to the Sudakov logarithms they do not factorize and exponentiate.

The leading-order coefficient $C_{1}$ determined by the one-loop Feynman diagrams given in Fig.1 is well-known and can be extracted from the full result for the $g g \rightarrow H g$ amplitude [25]. In Ref. [1] we have computed the two-loop coefficient $C_{2}$ determined by the Feynman diagrams in Fig.2 and generalized this result to $C_{n}$ with arbitrary $n$. In the next section we present the expression for the corresponding corrections to the partonic cross section and discuss their numerical impact on the Higgs boson transverse momentum distribution.

\section{Abelian double logarithmic corrections to the differential cross section}

The largest effect of the bottom quark on the differential cross section is caused by its interference with the top quark contribution. Note that since the leading bottom quark effect is due to the interference with the top quark mediated amplitude, to leading order in $1 / m_{t}$, any additional real emission contribution involves the three-gluon interaction and does not contribute to the abelian part of the correction. It is convenient to express the correction to the cross section through the variables $\tau=\ln \left(m_{b}^{2} / p_{\perp}^{2}\right) / L$ and $\zeta=\ln (u / t) / L$ with $L=\ln \left(s / m_{b}^{2}\right)$, which parameterize the dependence of the cross section on the transverse momentum and rapidity. Neglecting the finite top mass effects in the interference term we obtain [1]

$$
d \sigma_{g g \rightarrow H g}=d \sigma_{g g \rightarrow H g}^{(0)} \times\left[1-\frac{3}{2} \frac{m_{b}^{2}}{M_{H}^{2}} L^{2} f(x, \tau, \zeta)+\mathscr{O}\left(m_{b}^{4}\right)\right],
$$

where

$$
x f(x, \tau, \zeta)=\int_{0}^{1} \frac{\mathrm{d} \eta}{\eta}\left[\left(1-e^{-x \eta(1-\eta)}\right)(1+2 \theta(1-\tau-\zeta-2 \eta))-\left(1-e^{-x \eta \delta(\tau, \zeta)}\right)\right]
$$




$$
+e^{-x \delta(\tau, \zeta)} \int_{(1-\tau+\zeta) / 2}^{(1+\tau+\zeta) / 2} \frac{\mathrm{d} \eta}{\eta}\left(1-e^{-x \eta(1+\tau+\zeta-2 \eta) / 2}\right)+(\zeta \rightarrow-\zeta)
$$

$x=\frac{C_{F} \alpha_{s}}{2 \pi} L^{2}$ is the double logarithmic expansion parameter, and $\delta(\tau, \zeta)=\left((1-\tau)^{2}-\zeta^{2}\right) / 4$. The perturbative expansion of the function $f$ reads

$$
f=2-\frac{x}{6}\left(1-\tau^{3}+\tau^{4}\right)+\frac{x^{2}}{24}\left(\frac{4}{15}-\tau^{3}+2 \tau^{4}-\frac{7 \tau^{5}}{5}+\frac{2 \tau^{6}}{5}+\zeta^{2}\left(\tau^{3}-\tau^{4}\right)\right)+\ldots
$$

where ellipsis stands for terms suppressed by higher powers of $x$.

We can use the result Eq.(3.3) to estimate the impact of the QCD corrections to bottom quark contributions to $\mathrm{gg} \rightarrow \mathrm{Hg}$ on the Higgs boson transverse momentum distribution. In principle, we should convolute the partonic cross section Eq.(3.1) with the parton distribution functions. However, we will now argue that, given the structure of the corrections shown in Eq.(3.3), this is not necessary. Indeed, within the accuracy of our approximation $L=\ln \left(s / m_{b}^{2}\right) \approx \ln \left(m_{H}^{2} / m_{b}^{2}\right)$ can be considered independent of the partonic center-of-mass energy. In addition, the series in Eq.(3.3) shows very weak dependence on the rapidity of the soft gluon. Indeed, the function $f$ in Eq.(3.3) does not depend on the gluon rapidity up to $\mathscr{O}(x)$. Moreover, at $\mathscr{O}\left(x^{2}\right)$ the rapidity-dependent part of the coefficient includes only high powers of $\tau$. If the soft gluon is emitted at large rapidity, $|\zeta| \approx 1$ and $\tau \ll 1$. On the contrary, central emission with the large transverse momentum implies $|\zeta| \ll 1$ and $\tau \approx 1$. Therefore, the rapidity-dependent term is small everywhere and can be neglected. After these modifications the function $f$ depends only on the transverse momentum of the emitted gluon or the Higgs boson. As a result it remains unaffected by the integration over parton distribution functions if the transverse momentum of the Higgs boson is kept fixed. Therefore, we can write

$$
\begin{aligned}
\frac{d \sigma_{p p \rightarrow H+j}}{d p_{\perp}^{2}} & =\frac{d \sigma_{p p \rightarrow H+j}^{(0)}}{d p_{\perp}^{2}}\left\{1-\frac{3 m_{b}^{2}}{m_{H}^{2}} L_{\mathrm{eff}}^{2}\left[1-\frac{x_{\mathrm{eff}}}{12}\left(1-\tau^{3}+\tau^{4}\right)\right.\right. \\
& \left.\left.+\frac{x_{\mathrm{eff}}^{2}}{48}\left(\frac{4}{15}-\tau^{3}+2 \tau^{4}-\frac{7 \tau^{5}}{5}+\frac{2 \tau^{6}}{5}\right)+\mathscr{O}\left(x_{\mathrm{eff}}^{3}\right)\right]+\mathscr{O}\left(m_{b}^{4}\right)\right\}
\end{aligned}
$$

where $L_{\text {eff }}=\ln \left(m_{H}^{2} / m_{b}^{2}\right)$ and $x_{\text {eff }}=\frac{\alpha_{s} C_{F}}{2 \pi} L_{\text {eff }}^{2}$. We emphasize that Eq.(3.4) only applies to the contribution of $g g$ partonic channel to the production of the Higgs boson in proton collisions and that only abelian corrections are taken into account there.

We note that the series in Eq.(3.4) has peculiar structure. Indeed, the one-loop double logarithmic correction to $\mathrm{d} \sigma / \mathrm{d} p_{\perp}$ is independent on $p_{\perp}$, thanks to a cancellation between $p_{\perp}$-dependent contributions to individual helicity amplitudes, when the differential cross section is evaluated [21]. In principle, it could have been possible to interpret this result as an indication that the naive factorization of soft emissions extends to a region beyond $p_{\perp}>m_{b}$, at least inasmuch as the interference with the top quark loop is concerned. However, our result Eq.(3.4) shows that such an interpretation does not hold and that the cancellation of $p_{\perp}$-dependent double logarithmic corrections does not persist beyond one-loop. In fact, starting from three loops, the double logarithmic corrections to the differential cross section start to depend on the rapidity of the emitted gluon as Eq.(3.3) shows.

To understand the numerical impact of these corrections, we use $m_{H}=125 \mathrm{GeV}, m_{b}=$ $4.2 \mathrm{GeV}, \alpha_{s}=0.12$ and consider $p_{\perp}$ in the range $m_{b}<p_{\perp}<50 \mathrm{GeV}$. We note that the oneloop double logarithmic corrections reduce the cross section by about $16 \%$. This is somewhat 


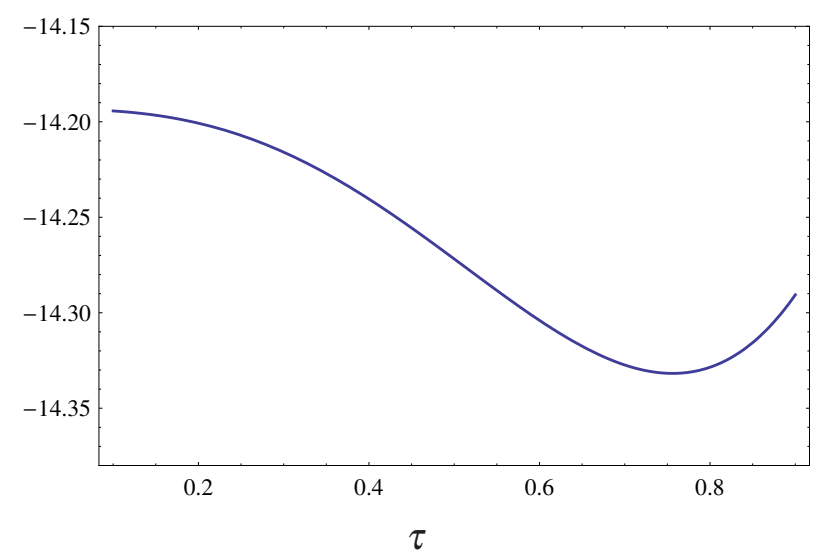

Figure 3: The bottom-quark loop corrections to the Higgs boson transverse momentum distribution, Eq.(3.4), in percent to the leading top-quark loop contribution as function of the variable $\tau=$ $\ln \left(m_{b}^{2} / p_{\perp}^{2}\right) / \ln \left(m_{b}^{2} / m_{h}^{2}\right)$. The values of the input parameters are specified in the text. The transverse momentum dependence of the corrections is numerically dominated by the two-loop term.

larger than the result of the full computation, but still in the right ballpark. The two-loop correction increases the result by about $1.5 \%$. This is somewhat smaller than the next-to-leading order effect in $g g \rightarrow H$ cross section but, given the fact that we only consider the abelian contribution here, the two results are not inconsistent. ${ }^{4}$ However, our main interest is in $p_{\perp}$-dependent corrections and these corrections turn out to be quite small, see Fig.3. In fact, the two-loop correction in Eq.(3.4) decreases by just about $0.2 \%$ when the transverse momentum varies from $p_{\perp} \sim m_{b}$ to $p_{\perp} \sim 50 \mathrm{GeV}$. This tiny change is the result of a strong cancellation between $\tau^{3}$ and $\tau^{4}$ term in Eq.(3.4). When taken separately, these terms could have caused a change in the two-loop result that is closer to one percent. The three-loop correction in Eq.(3.4) changes the prediction by about $-0.1 \%$ and its $p_{\perp}$-dependent part is one order of magnitude smaller.

\section{Conclusion}

In this paper, we have studied the bottom-quark loop contribution to the production of the Higgs boson in association with a jet in gluon fusion in the double logarithmic approximation. This contribution is suppressed by the ratio of the bottom-quark mass to the Higgs boson mass but, at the same time, it is enhanced by two powers of large logarithms, $\ln \left(s / m_{b}^{2}\right)$ or $\ln \left(p_{\perp}^{2} / m_{b}^{2}\right)$, per one power of the strong coupling constant. As it is repeatedly emphasized in the literature, these terms may be important for phenomenology, in particular for the description of the Higgs boson transverse momentum distribution in an interesting kinematic region $m_{b}<p_{\perp}<m_{H}$. We have analyzed the abelian part of the double logarithmic corrections and computed the $g g \rightarrow H g$ helicity amplitudes which incorporate these terms to all orders in $\alpha_{s}$.

Numerically, the abelian corrections appear to be moderate. For example, the two-loop corrections change the transverse momentum distribution by about two percent. However it is important

\footnotetext{
${ }^{4}$ The top-bottom interference changes the $m_{t} \rightarrow \infty$ inclusive cross section by approximately $-12 \%$ at leading order. QCD corrections to the bottom loop decrease this leading order result by fifty percent.
} 
to note that the $p_{\perp}$-dependent part of these corrections is only about $0.2 \%$ due to the cancellation between different $p_{\perp}$-dependent terms. Assuming that, up to an obvious change in the color factor $C_{F} \rightarrow C_{A}$, the non-abelian corrections will be similar to the abelian ones, we estimate the yet unknown non-abelian corrections to be about three times larger. We conclude that the description of the Higgs boson transverse momentum distribution with a few percent precision requires a calculation of the $\mathscr{O}\left(\alpha_{s}\right)$ logarithmically enhanced non-abelian corrections to bottom quark contribution while the all-order resummation is, probably, not important. Our analysis sets up a framework for such a calculation. A new element in the calculation of the non-abelian part is its infra-red sensitivity and a related need to account for the contribution of soft radiation.

\section{References}

[1] K. Melnikov and A. Penin, JHEP 1605 (2016) 172.

[2] G. Aad et al. [ATLAS Collaboration], Phys. Lett. B 716 (2012) 1.

[3] S. Chatrchyan et al. [CMS Collaboration], Phys. Lett. B 716 (2012) 30.

[4] C. Anastasiou, C. Duhr, F. Dulat, F. Herzog and B. Mistlberger, Phys. Rev. Lett. 114 (2015) 212001.

[5] C. Arnesen, I. Z. Rothstein and J. Zupan, Phys. Rev. Lett. 103 (2009) 151801.

[6] E. Bagnaschi, G. Degrassi, P. Slavich and A. Vicini, JHEP 1202 (2012) 088.

[7] A. Azatov and A. Paul, JHEP 1401 (2014) 014.

[8] E. Bagnaschi and A. Vicini, JHEP 1601 (2016) 056.

[9] U. Langenegger, M. Spira and I. Strebel, arXiv:1507.01373 [hep-ph].

[10] R. Boughezal, F. Caola, K. Melnikov, F. Petriello and M. Schulze, Phys. Rev. Lett. 115 (2015) 082003.

[11] R. Boughezal, C. Focke, W. Giele, X. Liu and F. Petriello, Phys. Lett. B 748 (2015) 5.

[12] D. de Florian, G. Ferrera, M. Grazzini and D. Tommasini, JHEP 1111 (2011) 064.

[13] T. Becher, M. Neubert and D. Wilhelm, JHEP 1305 (2013) 110.

[14] D. Neill, I. Z. Rothstein and V. Vaidya, JHEP 1512 (2015) 097.

[15] R. Mueller and D. G. Oeztuerk, arXiv:1512.08570 [hep-ph].

[16] R. Frederix, S. Frixione, E. Vryonidou and M. Wiesemann, arXiv:1604.03017 [hep-ph].

[17] F. Caola, S. Forte, S. Marzani, C. Muselli and G. Vita, arXiv:1606.04100 [hep-ph].

[18] V. V. Sudakov, Sov. Phys. JETP 3 (1956) 65, [Zh. Eksp. Teor. Fiz. 30 (1956) 87].

[19] H. Mantler and M. Wiesemann, Eur. Phys. J. C 73 (2013) 2467.

[20] M. Grazzini and H. Sargsyan, JHEP 1309 (2013) 129.

[21] A. Banfi, P. F. Monni and G. Zanderighi, JHEP 1401 (2014) 097.

[22] V. G. Gorshkov, V. N. Gribov, L. N. Lipatov and G. V. Frolov, Sov. J. Nucl. Phys. 6 (1968) 95, [Yad. Fiz. 6 (1967) 129].

[23] M. I. Kotsky and O. I. Yakovlev, Phys. Lett. B 418 (1998) 335.

[24] A. A. Penin, Phys. Lett. B 745 (2015) 69, [Corrigendum: Phys. Lett. B 751 (2015) 596].

[25] U. Baur and E. W. N. Glover, Nucl. Phys. B 339 (1990) 38. 